\title{
A Retrospective Cohort Study to Investigate the Incidence of Cachexia During Chemotherapy in Patients with Colorectal Cancer
}

\author{
Masayuki Shibata · Masaru Fukahori · Eiji Kasamatsu • \\ Koji Machii · Satoshi Hamauchi
}

Received: June 26, 2020 / Accepted: September 26, 2020 / Published online: October 16, 2020

(C) The Author(s) 2020

\begin{abstract}
Introduction: This retrospective study focused on cancer cachexia in clinical practice. We evaluated the incidence of cancer cachexia and the relationship between cancer cachexia and overall survival (OS) or toxicities in patients with advanced colorectal cancer after undergoing first-line systemic chemotherapy.

Methods: We examined 150 patients with colorectal cancer who underwent first-line systemic chemotherapy between February 1, 2010 and August 31, 2016 at Shizuoka Cancer Center Hospital and Kurume University Hospital. Cancer cachexia was defined as $>5 \%$ weight loss or $>2 \%$ weight loss with a body mass index of $<20 \mathrm{~kg} / \mathrm{m}^{2}$ within the past 6 months
\end{abstract}

Electronic Supplementary Material The online version of this article (https://doi.org/10.1007/s12325020-01516-6) contains supplementary material, which is available to authorized users.

M. Shibata $\cdot$ S. Hamauchi $(\square)$

Division of Gastrointestinal Oncology, Shizuoka

Cancer Center, Shizuoka, Japan

e-mail: s.hamauchi@scchr.jp

M. Fukahori

Multidisciplinary Treatment Cancer Center,

Kurume University Hospital, Fukuoka, Japan

E. Kasamatsu · K. Machii

Medical Affairs Department, Ono Pharmaceutical

Co., Ltd., Osaka, Japan according to the European Palliative Care Research Collaborative criteria.

Results: One hundred patients from Shizuoka Cancer Center and 50 from Kurume University Hospital were registered. Median age and body mass index were 65 years (range 29-85) and $21.7 \mathrm{~kg} / \mathrm{m}^{2}$ (14.8-32.5), respectively. Cumulative incidence of cancer cachexia was $50.7 \%$ at 24 weeks, and reached $91.3 \%$ over the whole study period. OS was significantly different between patients with and without cancer cachexia within 24 weeks after starting first-line treatment, although the onset of cancer cachexia within 24 weeks could not be considered as an independent prognostic factor for OS. Severe appetite loss and fatigue tended to occur more frequently in patients with cancer cachexia within 24 weeks.

Conclusion: Cancer cachexia appears to have an onset in approximately half of patients with advanced colorectal cancer within 24 weeks after starting first-line treatment. Although causal relationships were controversial, the onset of cancer cachexia within 24 weeks tends to be related to worse outcomes. Thus, it would be better to monitor weight loss leading to cachexia in patients with advanced colorectal cancer, especially within 24 weeks after starting first-line chemotherapy.

Trial Registration: University Hospital Medical Information Network Clinical Trials Registry (UMIN000035002). 
Keywords: Adverse events; Cancer cachexia; Colorectal cancer; Incidence; Survival

\section{Key Summary Points}

Why carry out this study?

In patients with advanced colorectal cancer after starting chemotherapy, there are few reports to investigate prevalence and onset of cancer cachexia based on the criteria of the European Palliative Care Research Collaborative.

We evaluated the incidence of cancer cachexia and the relationship between cancer cachexia and overall survival or toxicities in patients with advanced colorectal cancer after undergoing firstline systemic chemotherapy.

\section{What was learned from this study?}

Cancer cachexia appears to have an onset in approximately half of patients with advanced colorectal cancer within 24 weeks after starting first-line treatment, which likely results in poor prognosis.

In advanced colorectal cancer, severe appetite loss and fatigue tended to occur more frequently in patients with cancer cachexia than in patients without it.

\section{DIGITAL FEATURES}

This article is published with digital features, including a summary slide, to facilitate understanding of the article. To view digital features for this article, go to https://doi.org/10.6084/ m9.figshare.13007864.

\section{INTRODUCTION}

Upon progression, colorectal cancer causes severe symptoms including diarrhea, constipation, bloody stool, and weight loss. Colorectal cancer has recently become the most common cancer in Japan. For metastatic colorectal cancer, combinations of fluoropyrimidines, oxaliplatin, irinotecan, and biologic agents such as bevacizumab are recommended as first-line chemotherapy.

Patients with cancer often report cancer cachexia, which is a complex metabolic disorder predominantly characterized by body weight loss [1]. In 2011, the European Palliative Care Research Collaborative (EPCRC) proposed three diagnostic criteria for cancer cachexia: (1) $>5 \%$ weight loss in the past 6 months, (2) weight loss of $>2 \%$ and a body mass index (BMI) of $<20 \mathrm{~kg} / \mathrm{m}^{2}$ in the past 6 months, or (3) weight loss of $>2 \%$ and diagnosis of sarcopenia in the past 6 months [2]. It is clear that cancer cachexia can reduce the activities of daily living and lead to a poor quality of life (QOL) [1] due to progressive weakness of skeletal muscles. In addition, cancer cachexia and its associated metabolic changes may negatively affect the patient's ability to tolerate their cancer therapies, especially cytotoxic chemotherapy [3-9].

In patients with advanced non-small cell lung cancer (NSCLC), Takayama et al. examined the incidence of cancer cachexia that developed in each observation period of 4-12 weeks, 16-24 weeks, 28-36 weeks, and 40-52 weeks after the start of chemotherapy [10]. The prevalence of cancer cachexia as defined by EPCRC was similar in all observation periods. The study also reported that the patients with cancer cachexia tended to have a reduced QOL and shortened survival. In patients with pancreatic ductal adenocarcinoma, cancer cachexia during chemotherapy occurred in 32\% within 12 weeks of starting first-line chemotherapy, reaching $64 \%$ at 1 year [11]. Although the overall survival (OS) was not significantly different between patients with and without cancer cachexia during chemotherapy, some adverse events (AEs) tended to be more frequent in patients with cancer cachexia. In patients with advanced gastric cancer, cancer cachexia was especially observed in more than half of the patients within 12 weeks after starting chemotherapy, and the cancer cachexia appeared to relate to the severity of AEs or reduced survival [12]. 
In advanced colorectal cancer, on the other hand, few reports have investigated the prevalence and onset of cancer cachexia based on EPCRC criteria in patients after starting chemotherapy, whereas incidences of cancer cachexia before chemotherapy or during palliative care, and the correlations between cancer cachexia before chemotherapy and prognosis, toxicity, and QOL have been reported previously [13-19].

Accordingly, we performed this retrospective study to investigate the incidence, characteristics, and outcomes of cancer cachexia after the start of first-line chemotherapy in patients who had been diagnosed with advanced colorectal cancer with local progression or distant metastasis in Japan. Expected findings should provide new clinical insights into cancer cachexia affected by not only the cancer but also the chemotherapy, which may identify when more careful observation is required during chemotherapy for patients with colorectal cancer.

Cancer cachexia was defined by the above EPCRC criteria (1) and (2). We did not use the third criterion because sarcopenia was not diagnosed in daily clinical practice and there were few records during observation periods. In this study, we also evaluated the relationships between cancer cachexia and the incidence of AEs, OS, treatment status, and laboratory values to clarify the influence of cancer cachexia on patients with colorectal cancer receiving cancer chemotherapy.

\section{METHODS}

\section{Ethics}

This study was approved in advance by the ethics review committee of Shizuoka Cancer Center (reference T30-19-30-1-7) and the results were reviewed by an ethics review committee of Kurume University Hospital (reference 18234). This study was registered on the University Hospital Medical Information Network Clinical Trials Registry (UMIN000035002).

\section{Patients}

Medical records databases were searched to retrieve the records of patients who were diagnosed with advanced colorectal cancer with local progression or distant metastasis and who underwent first-line systemic chemotherapy for advanced colorectal cancer between February 1, 2010 and August 31, 2016 at Shizuoka Cancer Center Hospital and Kurume University Hospital.

\section{Definition of Cancer Cachexia}

We defined cancer cachexia as either (1) weight loss of $>5 \%$ in the past 6 months or (2) weight loss of $>2 \%$ in the past 6 months with BMI of $<20 \mathrm{~kg} / \mathrm{m}^{2}$. The start date of chemotherapy was recorded as the index date until the first 6 months after first-line treatment.

Data for these two cachexia criteria (1 and 2) are the most available in clinical practice of the three criteria proposed by the EPCRC [2]. However, the third criterion (weight loss of $>2 \%$ with sarcopenia within the past 6 months) was not set as cancer cachexia, because records regarding sarcopenia were available for only a limited number of the patients during observational periods. As Antoun et al. reported that $87.4 \%$ of patients with cancer cachexia were diagnosed on the basis of two criteria (1 and 2), we expected to be able to evaluate most of the patients with cancer cachexia in this study [20].

\section{Data Collection}

The data for body weight, laboratory tests, and AEs (classified according to Common Terminology Criteria for Adverse Events version 5.0) were collected at the start of chemotherapy (0 weeks) and during observation periods. The lowest body weight every 4 weeks, the latest laboratory test, and the worst $\mathrm{AE}$ data at each observation period $(0,1-12,13-24,25-36$, and $37-48$ weeks, and $>48$ weeks) were collected. 


\section{Data Analyses}

Primary endpoints were the incidence and timing to onset $(0-12,13-24,25-36$, and 37-48 weeks, and $>48$ weeks) of cancer cachexia and the cumulative incidence of cancer cachexia from the start of first-line chemotherapy up to 156 weeks.

Secondary endpoints were the relationships between cancer cachexia and the incidence of AEs, OS, treatment status, and laboratory values.

OS was calculated from the beginning of first-line chemotherapy and was assessed using the Kaplan-Meier method. OS was compared using the log-rank test between patients who showed cancer cachexia within 12, 24, and 48 weeks of starting first-line chemotherapy and patients without cachexia. All $p$ values were two-sided, and $p<0.05$ was considered statistically significant. We originally planned to analyze data according to the presence or absence of cancer cachexia within 48 weeks of starting first-line treatment. Owing to the disparity in the number of patients with or without cancer cachexia at this time point, we also performed exploratory analyses in which patients were divided according to their experiences of cancer cachexia within 12 weeks or 24 weeks of starting first-line chemotherapy.

Hazard ratios and 95\% confidence intervals (CIs) of presence or absence of cancer cachexia for OS were evaluated using a Cox proportional hazards model with or without adjusted model stratified by neutrophil/lymphocyte ratio, BMI, carcinoembryonic antigen (CEA), carbohydrate antigen 19-9, presence of liver metastases, and Eastern Cooperative Oncology Group Performance Status (ECOG PS). The cutoff period for occurrence of cancer cachexia was 24 weeks, because the number of patients was similar in both groups. Median survival times were calculated with 95\% CIs, which were determined using the Brookmeyer and Crowley method.

The incidence, timing, and grade of appetite loss and fatigue in cancer cachexia were investigated since appetite loss and fatigue were reported as the most relevant AEs in cancer cachexia in the previous study [10].
Incidences of cancer cachexia up to 12 weeks and 156 weeks were calculated for patient subpopulations stratified by response to initial chemotherapy, ECOG PS, and primary site in patient background characteristics.

Data analyses were performed using SAS for Windows version 9.4 or later (SAS Institute, Cary, NC, USA).

\section{RESULTS}

\section{Patients}

A total of 150 patients (90 men and 60 women) were registered in this study (Table 1). Median age and BMI were 65 years (range 29-85) and $21.7 \mathrm{~kg} / \mathrm{m}^{2}$ (14.8-32.5), respectively. The proportions of patients with ECOG PSO, PS1, and PS2 were $50.0 \%, 43.4 \%$, and $6.0 \%$, respectively. Primary tumor sites were rectum (60.0\%) and colon (40.0\%), and most patients had stage IV cancer (99.3\%). First-line chemotherapy was $\mathrm{XELOX/FOLFOX/SOX} \pm$ bevacizumab (BV) (49.3\%), FOLFIRI/IRIS/irinotecan (CPT11) \pm BV (18.0\%), FOLFOXIRI \pm BV (6.0\%), capecitabine/S-1/FL (5-fluorouracil + leucovorin) $\pm \mathrm{BV} \quad(6.7 \%), \quad$ FOLFIRI/CPT-11 + cetuximab/panitumumab (4.7\%), and FOLFOX + cetuximab/panitumumab (14.7\%). The medians of neutrophil/lymphocyte ratio, C-reactive protein (CRP), albumin, and hemoglobin were 3.37 (0.74-16.17), $0.995 \mathrm{mg} / \mathrm{l}$ (0.04-19.18), $3.70 \mathrm{~g} / \mathrm{dl}(1.70-4.90)$, and $11.8 \mathrm{~g} /$ dl (7.5-16.7), respectively.

\section{Incidence and Timing of Cancer Cachexia}

Figure $1 \mathrm{a}$ shows the incidence and timing of cancer cachexia onset $(1-12,13-24,25-36$, and $37-48$ weeks, and > 48 weeks); $42.7 \%$ of patients experienced cancer cachexia within 12 weeks after starting first-line chemotherapy. Cancer cachexia occurred during their first-line chemotherapy in 91 patients $(60.7 \%)$.

Figure $1 \mathrm{~b}$ shows the cumulative incidence of cancer cachexia after the start of first-line chemotherapy, which was $50.7 \%$ at 24 weeks, 
Table 1 Patient characteristics

\begin{tabular}{|c|c|}
\hline Characteristic & Patient $N=150$ \\
\hline \multicolumn{2}{|l|}{ Sex } \\
\hline Male & $90(60.0)$ \\
\hline Female & $60(40.0)$ \\
\hline Age, years & $65.0(29-85)$ \\
\hline BMI, $\mathrm{kg} / \mathrm{m}^{2}$ & $21.72(14.8-32.5)$ \\
\hline \multicolumn{2}{|l|}{ ECOG PS } \\
\hline 0 & $75(50.0)$ \\
\hline 1 & $65(43.3)$ \\
\hline 2 & $9(6.0)$ \\
\hline 3 & $1(0.7)$ \\
\hline \multicolumn{2}{|l|}{ Primary site } \\
\hline Rectum & $90(60.0)$ \\
\hline Colon & $60(40.0)$ \\
\hline \multicolumn{2}{|l|}{ UICC stage } \\
\hline III & $1(0.7)$ \\
\hline IV & $149(99.3)$ \\
\hline \multicolumn{2}{|l|}{ Modified Glasgow prognosis score } \\
\hline $\begin{array}{l}\text { Albumin } \geq 3.5 \mathrm{~g} / \mathrm{dl} \text { and } \\
\mathrm{CRP}<1.0 \mathrm{mg} / \mathrm{dl}\end{array}$ & $67(44.7)$ \\
\hline $\begin{array}{l}\text { Albumin }<3.5 \mathrm{~g} / \mathrm{dl} \text { and } \\
\mathrm{CRP}<1.0 \mathrm{mg} / \mathrm{dl}\end{array}$ & $9(6.0)$ \\
\hline $\begin{array}{l}\text { Albumin } \geq 3.5 \mathrm{~g} / \mathrm{dl} \text { and } \\
\mathrm{CRP} \geq 1.0 \mathrm{mg} / \mathrm{dl}\end{array}$ & $31(20.7)$ \\
\hline $\begin{array}{l}\text { Albumin }<3.5 \mathrm{~g} / \mathrm{dl} \text { and } \\
\mathrm{CRP} \geq 1.0 \mathrm{mg} / \mathrm{dl}\end{array}$ & $43(28.7)$ \\
\hline \multicolumn{2}{|l|}{ First-line chemotherapy } \\
\hline $\mathrm{XELOX} / \mathrm{FOLFOX} / \mathrm{SOX} \pm \mathrm{BV}$ & $74(49.3 \%)$ \\
\hline FOLFIRI/IRIS/CPT- $11 \pm \mathrm{BV}$ & $27(18.0 \%)$ \\
\hline FOLFOXIRI $\pm \mathrm{BV}$ & $9(6.0 \%)$ \\
\hline Cape/S-1/FL $(5 F U+L V) \pm B V$ & $10(6.7 \%)$ \\
\hline $\begin{array}{l}\text { FOLFIRI/CPT-11 + Cmab/ } \\
\text { Pmab }\end{array}$ & $7(4.7 \%)$ \\
\hline FOLFOX $+\mathrm{Cmab} / \mathrm{Pmab}$ & $22(14.7 \%)$ \\
\hline
\end{tabular}

Table 1 continued

\begin{tabular}{lc}
\hline Characteristic & Patient $N=\mathbf{1 5 0}$ \\
\hline $\mathrm{CrCl}^{\mathrm{a}}, \mathrm{ml} / \mathrm{min}^{2}$ & 81.238 \\
& $(22.59-207.03)$ \\
Neutrophil/lymphocyte ratio & $3.37(0.74-16.17)$ \\
CA19-9, ng/ml & 94.00 \\
& $(1.0-32,965.0)$ \\
CEA, ng/ml & 112.35 \\
& $(0.8-152,905.9)$ \\
Sodium, mg/dl & $139.0(129-145)$ \\
Potassium, mg/dl & $4.30(3.5-5.6)$ \\
AST, U/l & $27.0(11-175)$ \\
ALT, U/l & $21.0(7-157)$ \\
ALP, U/l & $326.5(118-2603)$ \\
Total bilirubin, mg/dl & $0.500(0.20-3.00)$ \\
Neutrophil count, $/ \mu \mathrm{l}$ & 4907.0 \\
WBC count, $/ \mu \mathrm{l}$ & $(1270-11,611)$ \\
Lymphocyte count, $/ \mu \mathrm{l}$ & 7300.0 \\
Platelet count, $/ \mu \mathrm{l}$ & $(3510-17,000)$ \\
Creatinine, mg/dl & 1550.5 \\
Albumin, g/dl & $(593-3417)$ \\
HemP, mg/l & $28.65(10.0-60.7)$ \\
\hline & $0.690(0.33-1.78)$ \\
$3.700(1.70-4.90)$ \\
$0.995(0.04-19.18)$ \\
& $11.75(7.5-16.7)$ \\
\hline
\end{tabular}

Values are $n$ (\%) or median (range)

$B M I$ body mass index, ECOG PS Eastern Cooperative Oncology Group Performance Status, UICC Union for International Cancer Control, $B V$ bevacizumab, Cape capecitabine, $C P T-11$ irinotecan, 5-FU 5-fluorouracil, $L V$ leucovorin, $\mathrm{Cmab}$ cetuximab, $\mathrm{Pmab}$ panitumumab, $\mathrm{CrCl}$ creatinine clearance, CA19-9 carbohydrate antigen 19-9, $C E A$ carcinoembryonic antigen, $A S T$ aspartate aminotransferase, $A L T$ alanine aminotransferase, $A L P$ alkaline phosphatase, $W B C$ white blood cell count, $C R P$ C-reactive protein

${ }^{a}$ Cockcroft-Gault formula 
$65.3 \%$ at 48 weeks, and $91.3 \%$ over the whole study period.

Within 12 weeks after starting first-line chemotherapy, the incidence of cancer cachexia in patients with disease progression as their best overall response during first-line chemotherapy was higher than that in patients with stable disease/partial response (disease progression, $60.0 \%$; stable disease, $40.4 \%$; partial response, $42.4 \%)$. During 156 weeks, the incidence of cancer cachexia was $100 \%$ in the disease progression group, $87.2 \%$ in the stable disease group, and $93.5 \%$ in the partial response group (Fig. S1 in the electronic supplementary material).

Patients with ECOG PS $\geq 1$ had a higher incidence of cancer cachexia than patients with PS 0 within 12 weeks after starting first-line chemotherapy (PS $=0,33.3 \%$; PS $\geq 1,52.0 \%$ ), whereas the incidence within 156 weeks was comparable ( $\mathrm{PS}=0,89.3 \% ; \quad \mathrm{PS} \geq 1, \quad 93.3 \%$ ) (Fig. S2 in the electronic supplementary material).

Within 12 weeks, the incidence of cancer cachexia was higher in the group of patients whose primary tumor site was the colon than those in the rectum (colon, 54.4\%; rectum, $25.0 \%$ ), but at 156 weeks, the incidence in the colon group was similar to that in the rectum group (colon, 91.1\%; rectum, 91.7\%) (Fig. S3 in the electronic supplementary material).

The odds ratio (95\% CI) for the onset of cancer cachexia during first-line chemotherapy with regard to the response to first-line chemotherapy was $1.15(0.59,2.25)$ and the odds ratio with regard to disease control (response + stable disease) was $1.31(0.38,4.51)$. Thus, response or disease control during chemotherapy did not significantly correlate with onset of cancer cachexia (Table S1 in the electronic supplementary material).

\section{Survival}

Figure 2 shows OS for patients with or without cancer cachexia, within 12,24 , or 48 weeks after starting chemotherapy. Landmark analyses were also done at these time points. OS was significantly different between patients with and without cancer cachexia in the analysis at 24 weeks from the start of first-line chemotherapy (log-rank $p=0.0467$ ) (Fig. $2 \mathrm{a}$ ); the median survival time $(95 \% \mathrm{CI})$ was $720(570,820)$ days for patients with cancer cachexia versus 816 $(704,930)$ days for patients without cancer cachexia. However, in the landmark analysis at 24 weeks, a significant difference was not observed in the OS between patients with and without cancer cachexia (log-rank $p=0.0823$ ). Similarly, the difference was not observed when we classified patients according to experience of cancer cachexia within 12 weeks (Fig. 2b) or 48 weeks (Fig. 2c) after starting chemotherapy.

Hazard ratio $(95 \% \mathrm{CI})$ of presence or absence of cancer cachexia within 24 weeks of starting first-line chemotherapy was $1.38(0.96,1.99)$ for OS based on unadjusted models, and $1.12(0.75$, 1.67) for OS based on adjusted models. Neutrophil/lymphocyte ratio, CEA, and ECOG PS could be prognostic factors for survival in the adjusted model (Table 2).

\section{Treatment Status}

There were no appreciable differences in the percentages of patients who started second/ third-line chemotherapy between patients with and without cancer cachexia in the preceding line (data not shown).

\section{AEs in Patients with and Without Cancer Cachexia}

Figure 3 shows the incidence, timing, and grade of appetite loss and fatigue in patients with and without cancer cachexia. The rates of severe appetite loss and fatigue were apparently different between patients with and without cancer cachexia at 24 weeks. The rate of severe appetite loss in patients with cancer cachexia ( $\geq$ grade 2 ) was $5.3 \%$ at baseline (week 0), and reached $25.0 \%$ in the $0-48$ week period (Fig. 3a). In contrast, in the case of patients without cancer cachexia, the rates were $0 \%$ at baseline (week 0 ) and $9.5 \%$ in the $0-48$ week period (Fig. 3a). Regarding severe fatigue ( $\geq$ grade 2 ), the rate in the $0-48$ week period was higher in 
(a)

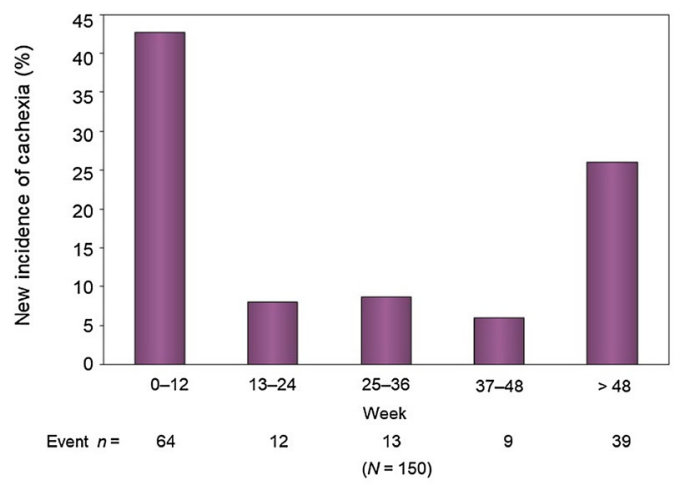

(b)

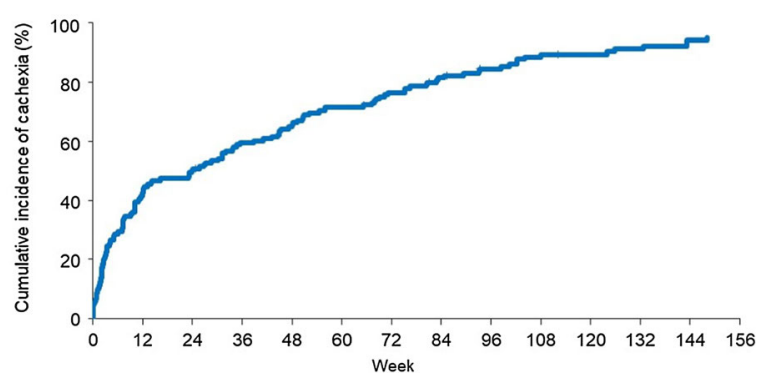

Fig. 1 a Timing of onset of cancer cachexia and $\mathbf{b}$ cumulative incidence of cancer cachexia

patients with cancer cachexia than in those without (Fig. 3b).

Cancer cachexia during chemotherapy was not significantly associated with appetite loss and fatigue during chemotherapy in univariate and multivariate analyses. On the other hand,
ECOG PS was significantly associated with appetite loss during chemotherapy [unadjusted and adjusted odds ratio (95\% CI) 3.56 (1.81, $6.97)$ and $3.36(1.70,6.63)$, respectively] but not with fatigue (Table $S 2$ in the electronic supplementary material).

\section{Laboratory Values}

Changes in laboratory test variables of neutrophil and lymphocyte counts, albumin, CRP, and hemoglobin were evaluated as representatives of cachexia. There appeared to be no difference in the changes in these values between patients with and without cancer cachexia within 24 weeks of starting first-line chemotherapy (Table 3).

\section{DISCUSSION}

The current retrospective study examined the occurrence of cancer cachexia in patients with advanced colorectal cancer after the start of first-line chemotherapy. The number of patients with cancer cachexia increased over time, and by the end of the study period, almost all patients showed cancer cachexia. Notably, about $40 \%$ of the patients had early onset cancer cachexia (by 12 weeks) after starting firstline chemotherapy. Similar early onsets were

Table 2 Prognostic factors for overall survival

$\mathrm{HR}^{\mathrm{a}}(95 \% \mathrm{CI})$

Presence of cancer cachexia within 24 weeks of starting treatment (yes vs. no)

$1.12(0.75-1.67)$

Neutrophil/lymphocyte ratio $(>3$ vs. $<3$ )

$1.92(1.27-2.90)$

BMI $\left(>28 \mathrm{~kg} / \mathrm{m}^{2}\right.$ vs. $\left.<28 \mathrm{~kg} / \mathrm{m}^{2}\right)$

$0.67(0.27-1.67)$

CEA $(>15 \mathrm{ng} / \mathrm{ml}$ vs. $<15 \mathrm{ng} / \mathrm{ml})$

$0.42(0.23-0.77)$

CA19-9 (> $50 \mathrm{ng} / \mathrm{ml}$ vs. $<50 \mathrm{ng} / \mathrm{ml})$

$1.13(0.73-1.75)$

Presence of liver metastases (yes vs. no)

$1.41(0.85-2.35)$

ECOG PS (2-3 vs. $0-1)$

$2.10(1.01-4.36)$

$H R$ hazard ratio, $C I$ confidence interval, $B M I$ body mass index, $C E A$ carcinoembryonic antigen, CA19-9 carbohydrate antigen 19-9, ECOG PS Eastern Cooperative Oncology Group Performance Status

${ }^{a}$ Hazard ratio in the multivalent Cox proportional hazard model analysis adjusted for the factors shown 
(a)

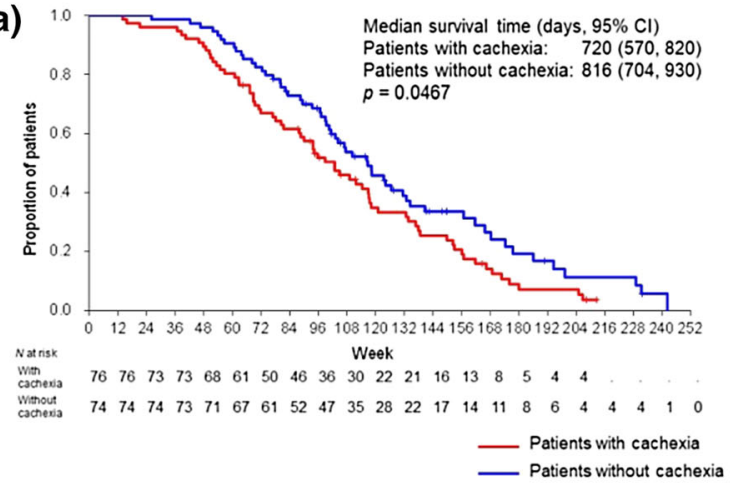

(c)

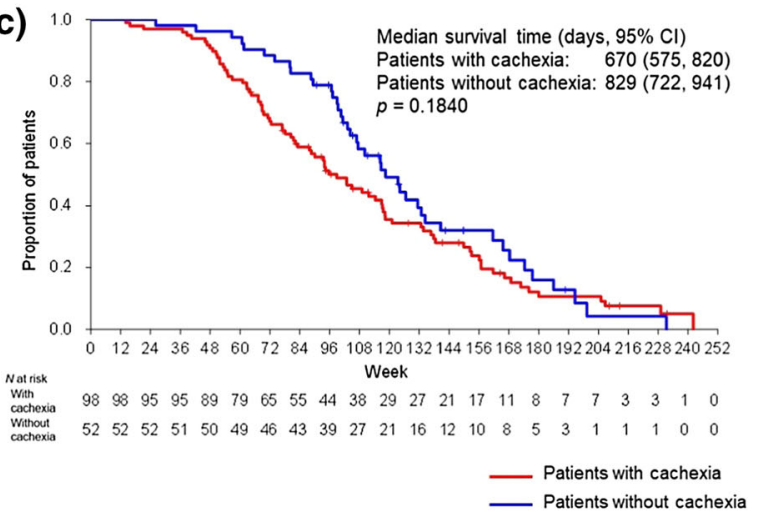

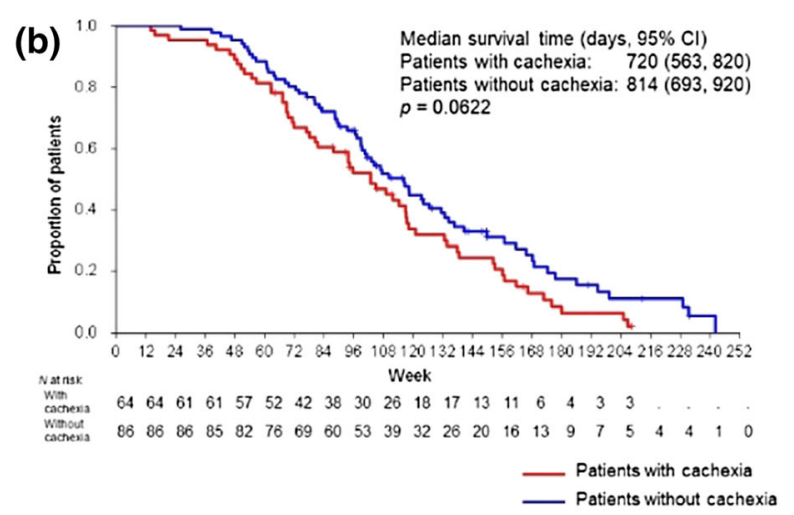

Fig. 2 Overall survival according to presence or absence of cancer cachexia within a 24 weeks, b 12 weeks, and $\mathbf{c} 48$ weeks after the start of chemotherapy. CI confidence interval

observed in patients with pancreatic ductal adenocarcinoma or advanced gastric cancer $[11,12]$. On the other hand, these trends appear to differ from that observed in patients with advanced NSCLC since the incidence of cancer cachexia was about $25 \%$ in this population at the early time point and was relatively constant over time [10]. These differences could be due to relative differences in response rates of first-line chemotherapy or essential differences of cancer, compared to patients with advanced NSCLC.

There was a significant difference in the OS at 24 weeks between patients with and without cancer cachexia. However, no difference was observed at other time points. Moreover, the onset of cancer cachexia within 24 weeks could not be considered as a prognostic factor for OS in either unadjusted or adjusted models. We also observed no difference in changes in laboratory values between patients with or without cancer cachexia recognized within 24 weeks.
Because this study permitted a number of different regimens for first-line chemotherapy, the type of chemotherapy may have had a varying impact on cancer cachexia, prognosis, and laboratory values. Indeed, previous studies have analyzed possible prognostic factors for patients with colorectal cancer treated with a specific single regimen, and found the neutrophil/lymphocyte ratio, and ECOG PS to be poor prognostic factors [21, 22]. The current study, which targeted multiple recent regimens used in daily clinical practice, also showed that the neutrophil/lymphocyte ratio and ECOG PS were poor prognostic factors. Therefore, the neutrophil/lymphocyte ratio and ECOG PS may affect the prognosis in colorectal cancer regardless of treatment regimen.

Although the incidence of cancer cachexia in patients with disease progression was relatively high compared to the stable disease/partial response group, there was no statistically 
(a)

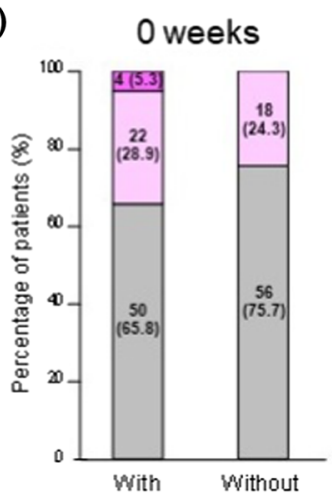

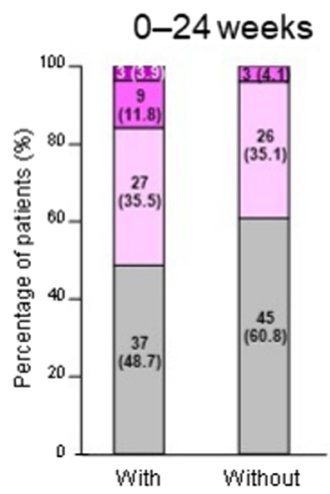

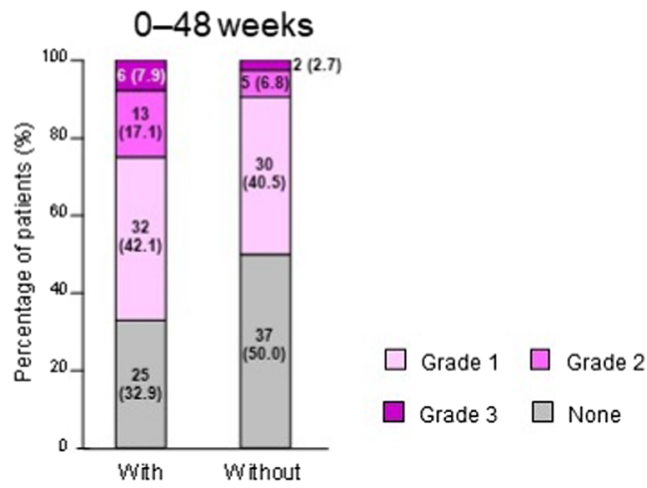

With: Presence of cancer cachexia within 24 weeks of starting chemotherapy Without: Absence of cancer cachexia within 24 weeks of starting chemotherapy

(b)

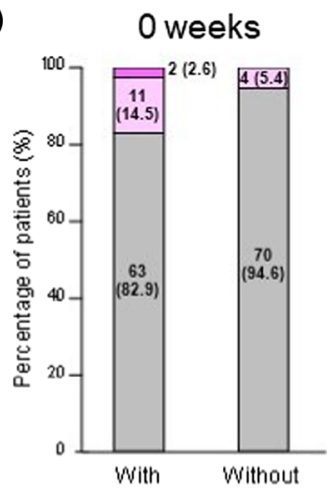

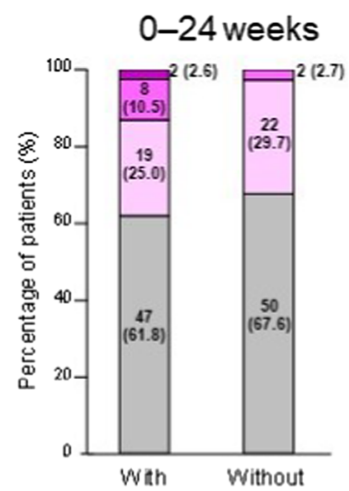

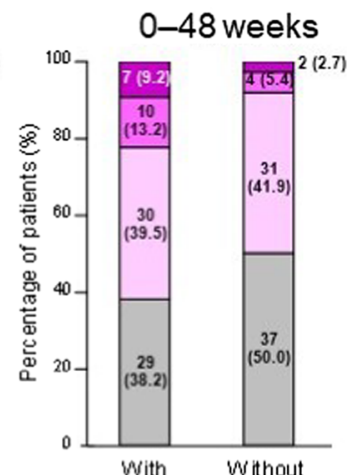

$\square$ Grade $1 \square$ Grade 2

Grade $3 \square$ None

With: Presence of cancer cachexia within 24 weeks of starting chemotherapy Without: Absence of cancer cachexia within 24 weeks of starting chemotherapy

Fig. 3 Appetite loss (a) and fatigue (b) in patients with or without cancer cachexia

significant correlation between the incidence of cancer cachexia during chemotherapy and responses or disease control. It is possible that weight loss due to reduced ascites resulting from successful chemotherapy may occur in the stable disease/partial response group. On the other hand, body weight may increase in some patients without response to chemotherapy even if they experience decreased appetite, and this may also be attributed to ascites. Therefore, in addition to a patient's body weight, any possible confounders, such as ascites, should be monitored during cancer therapy. Since individuals differ in terms of their build, lifestyle, dietary habits, and background metabolic rate, other conventional indicators such as inflammation markers may additionally be considered to define cancer cachexia in routine clinical practice.

The cumulative frequencies of AEs such as appetite loss and fatigue were increased regardless of cancer cachexia. In a multivariate analysis considering PS as the major confounder, cancer cachexia during chemotherapy was not significantly associated with appetite loss or fatigue during chemotherapy. Although we 
Table 3 Correlation between the presence or absence of cancer cachexia within 24 weeks after the start of first-line chemotherapy and the change from baseline in selected laboratory values

\begin{tabular}{|c|c|c|c|c|c|c|c|c|}
\hline & \multirow{2}{*}{$\begin{array}{l}n \\
-\end{array}$} & \multicolumn{3}{|c|}{ With cancer cachexia $(n=76)$} & \multirow{2}{*}{$\begin{array}{l}n \\
-\end{array}$} & \multicolumn{3}{|c|}{ Without cancer cachexia $(n=74)$} \\
\hline & & Median & Min & Max & & Median & Min & $\operatorname{Max}$ \\
\hline \multicolumn{9}{|c|}{$\mathrm{CRP}(\mathrm{mg} / \mathrm{dl})$} \\
\hline 12 weeks & 76 & -0.290 & -16.94 & 18.14 & 72 & -0.300 & -14.47 & 4.90 \\
\hline 24 weeks & 73 & -0.310 & -12.81 & 15.40 & 72 & -0.235 & -14.72 & 6.39 \\
\hline 48 weeks & 68 & -0.275 & -12.67 & 28.24 & 67 & -0.280 & -7.05 & 27.24 \\
\hline \multicolumn{9}{|c|}{ Albumin (g/dl) } \\
\hline 12 weeks & 76 & 0.02 & -2.0 & 1.1 & 73 & 0.10 & -1.2 & 1.3 \\
\hline 24 weeks & 73 & 0.10 & -1.1 & 1.3 & 74 & 0.02 & -1.2 & 1.7 \\
\hline 48 weeks & 68 & 0.10 & -1.9 & 1.4 & 68 & 0.00 & -1.2 & 2.0 \\
\hline \multicolumn{9}{|c|}{ Neutrophils $(/ \mu \mathrm{l})$} \\
\hline 12 weeks & 75 & -2263.0 & -9351 & 12,953 & 73 & -2538.0 & -8151 & 1831 \\
\hline 24 weeks & 73 & -2477.0 & $-10,673$ & 3562 & 73 & -2406.0 & -8334 & 4607 \\
\hline 48 weeks & 68 & -1735.5 & -9919 & 22,830 & 69 & -1647.0 & -6956 & 10,602 \\
\hline \multicolumn{9}{|c|}{ Total lymphocytes $(/ \mu \mathrm{l})$} \\
\hline 12 weeks & 76 & 93.0 & -990 & 1390 & 74 & 17.0 & -989 & 1246 \\
\hline 24 weeks & 73 & 79.0 & -1230 & 1194 & 74 & 135.0 & -1241 & 1425 \\
\hline 48 weeks & 68 & 100.0 & -1469 & 1309 & 69 & 40.0 & -1751 & 1497 \\
\hline
\end{tabular}

$C R P$ C-reactive protein

have not considered any other factors in this analysis, there are many possible confounders such as the biology of the illness and response to chemotherapy, which may affect correlation between these AEs and onset of cancer cachexia.

The accompanying AEs are of considerable concern because they may interfere with the continuation of chemotherapy and negatively affect the patient's well-being and QOL, although this study did not directly compare the cause-effect relationship between cancer cachexia and onset of these AEs.

The current study showed that cancer cachexia during chemotherapy may cause appetite loss and fatigue, and these AEs may be related to poor PS, suggesting that dietary intervention and nutritional counseling may be important for dealing with such poor PS and cancer cachexia during chemotherapy. Murphy et al. reported that nutritional intervention with $2.2 \mathrm{~g}$ of fish oil-derived eicosapentaenoic acid per day in patients with NSCLC appears to provide a benefit over standard of care, resulting in the maintenance of weight and muscle mass during first-line chemotherapy [23]. Mouri et al. reported the excellent feasibility of, and compliance with, multimodal intervention that included nutritional treatment during first-line chemotherapy in elderly patients with advanced cancer who were at risk for cancer cachexia [24]. These results also emphasize the importance of monitoring cancer cachexia, nutritional counseling, and nutritional intervention at the beginning of chemotherapy.

The EPCRC defines three diagnostic criteria for cancer cachexia as mentioned above. All 
include the degree of weight loss within the past 6 months, and two criteria comprise either concomitant low BMI or diagnosis of sarcopenia. Since sarcopenia is typically diagnosed by dual-energy X-ray absorptiometry based on the definition published by the European Working Group on Sarcopenia in Older People, the diagnosis method is not practical within an inpatient setting. In the present study, we applied cancer cachexia corresponding to the first two criteria of cancer cachexia without sarcopenia, because information on sarcopenia was available in only a limited number of the patients. Nevertheless, it is likely that most patients with cancer cachexia could be evaluated in the present study, because in another study, $87.4 \%$ of the patients with cachexia were diagnosed without the third criterion [20]. Because body weight measurement is easy for healthcare professionals to handle, information on cancer cachexia defined solely by body weight in clinical practice would be useful [25].

\section{Limitations}

There are limitations of this study including its retrospective design and inclusion criteria permitting a variety of first-line chemotherapy regimens. In addition, although we found a higher rate of AEs in the patients with cancer cachexia, due to the design of our study, we could not determine whether cancer cachexia contributes to the onset and grade of the AEs, or vice versa. Since this study was conducted as a retrospective study and clinical data were collected at only two institutions, a prospective study in multiple sites might be expected to yield further evidence to support the results of this study.

As mentioned above, we used two EPCRC criteria to define cancer cachexia.

\section{CONCLUSIONS}

Cancer cachexia appears to have an onset in approximately half of patients with advanced colorectal cancer within 24 weeks after starting first-line treatment. Although causal relationships were controversial, the onset of cancer cachexia within 24 weeks tends to be related to worse outcomes. Thus, it would be better to monitor weight loss leading to cachexia of patients with advanced colorectal cancer, especially within 24 weeks after starting first-line chemotherapy.

\section{ACKNOWLEDGEMENTS}

Funding. This study and the journal's Rapid Service and Open Access Fees were funded by Ono Pharmaceutical Co. Ltd.

Additional Assistance. The authors thank Koji Oba (The University of Tokyo) for his advice with statistical analysis. The authors also thank DOT WORLD Co., Ltd. for organization of the study, data collection, and statistical analysis; this was funded by Ono Pharmaceutical Co., Ltd.

Authorship. All named authors meet the International Committee of Medical Journal Editors (ICMJE) criteria for authorship for this article, take responsibility for the integrity of the work as a whole, and have given their approval for this version to be published.

Disclosures. Masayuki Shibata and Satoshi Hamauchi have received research funds from Ono Pharmaceutical Co., Ltd. Masaru Fukahori has received research funds from Ono Pharmaceutical Co., Ltd. and personal fees from Merck. Eiji Kasamatsu and Koji Machii are employees of Ono Pharmaceutical Co., Ltd.

Compliance with Ethics Guidelines. This study was approved in advance by the ethics review committee of Shizuoka Cancer Center (reference T30-19-30-1-7) and the results was reviewed by an ethics review committee of Kurume University Hospital (reference 18234). This study was registered on the University Hospital Medical Information Network Clinical Trials Registry (UMIN000035002).

Data Availability. Qualified researchers may request Ono Pharma to disclose individual 
patient-level data from clinical studies through the following website: https://www. clinicalstudydatarequest.com/. For more information on Ono Pharma's Policy for the Disclosure of Clinical Study Data, please see the following website: https://www.ono.co.jp/eng/ rd/policy.html.

Open Access. This article is licensed under a Creative Commons Attribution-NonCommercial 4.0 International License, which permits any non-commercial use, sharing, adaptation, distribution and reproduction in any medium or format, as long as you give appropriate credit to the original author(s) and the source, provide a link to the Creative Commons licence, and indicate if changes were made. The images or other third party material in this article are included in the article's Creative Commons licence, unless indicated otherwise in a credit line to the material. If material is not included in the article's Creative Commons licence and your intended use is not permitted by statutory regulation or exceeds the permitted use, you will need to obtain permission directly from the copyright holder. To view a copy of this licence, visit http://creativecommons.org/licenses/by$\mathrm{nc} / 4.0 /$.

\section{REFERENCES}

1. Baracos VE, Mazurak VC, Bhullar AS. Cancer cachexia is defined by an ongoing loss of skeletal muscle mass. Ann Palliat Med. 2019;8:3-12.

2. Fearon K, Strasser F, Anker SD, et al. Definition and classification of cancer cachexia: an international consensus. Lancet Oncol. 2011;12:489-95.

3. Bruggeman AR, Kamal AH, LeBlanc TW, Ma JD, Baracos VE, Roeland EJ. Cancer cachexia: beyond weight loss. J Oncol Pract. 2016;12:1163-71.

4. Escamilla DM, Jarrett P. The impact of weight loss on patients with cancer. Nurs Times. 2016;112: 20-2.

5. Lau SKM, Iyengar P. Implications of weight loss for cancer patients receiving radiotherapy. Curr Opin Support Palliat Care. 2017;11:261-5.
6. Tarricone R, Ricca G, Nyanzi-Wakholi B, MedinaLara A. Impact of cancer anorexia-cachexia syndrome on health-related quality of life and resource utilisation: a systematic review. Crit Rev Oncol Hematol. 2016;99:49-62.

7. Sasaki S, Oki E, Saeki H, et al. Skeletal muscle loss during systemic chemotherapy for colorectal cancer indicates treatment response: a pooled analysis of a multicenter clinical trial (KSCC 1605-A). Int J Clin Oncol. 2019;24:1204-13.

8. Blum D, Omlin A, Fearon K, et al. Evolving classification systems for cancer cachexia: ready for clinical practice? Support Care Cancer. 2010;18: 273-9.

9. Kimura M, Naito T, Kenmotsu H, et al. Prognostic impact of cancer cachexia in patients with advanced non-small cell lung cancer. Support Care Cancer. 2015;23:1699-708.

10. Takayama K, Atagi S, Imamura F, et al. Quality of life and survival survey of cancer cachexia in advanced non-small cell lung cancer patients-Japan nutrition and QOL survey in patients with advanced non-small cell lung cancer study. Support Care Cancer. 2016;24:3473-80.

11. Mitsunaga S, Kasamatsu E, Machii K. Incidence and frequency of cancer cachexia during chemotherapy for advanced pancreatic ductal adenocarcinoma. Support Care Cancer. 2020;28:5271-9.

12. Fukahori M, Shibata M, Hamauchi S, Kasamatsu E, Machii K. A retrospective cohort study to investigate the incidence of cancer-related weight loss during chemotherapy in gastric cancer patients. Support Care Cancer. 2020. https://doi.org/10. 1007/s00520-020-05479-w.

13. Thoresen L, Frykholm G, Lydersen S, et al. Nutritional status, cachexia and survival in patients with advanced colorectal carcinoma. Different assessment criteria for nutritional status provide unequal results. Clin Nutr. 2012;32:65-72.

14. Wallengren O, Lundholm K, Bosaeus I. Diagnostic criteria of cancer cachexia: relation to quality of life, exercise capacity and survival in unselected palliative care patients. Support Care Cancer. 2013;21:1569-77.

15. Hamauchi S, Furuse J, Takano T, et al. A multicenter, open-label, single-arm study of anamorelin (ONO-7643) in advanced gastrointestinal cancer patients with cancer cachexia. Cancer. 2019;125: 4294-302.

16. Kurk S, Peeters P, Stellato R, et al. Skeletal muscle mass loss and dose-limiting toxicities in metastatic 
colorectal cancer patients. J Cachex Sarcopenia Muscle. 2019;10:803-13.

17. Dewys WD, Begg C, Lavin PT, et al. Prognostic effect of weight loss prior to chemotherapy in cancer patients. Eastern Cooperative Oncology Group. Am J Med. 1980;69:491-7.

18. Miyamoto Y, Hiyoshi Y, Akiyama T, et al. Low skeletal muscle mass before salvage-line chemotherapy is a poor prognostic factor in patients with refractory metastatic colorectal cancer. Digestion. 2019;99:79-85.

19. Andreyev HJ, Norman AR, Oates J, Cunningham D. Why do patients with weight loss have a worse outcome when undergoing chemotherapy for gastrointestinal malignancies? Eur J Cancer. 1998;34: 503-9.

20. Antoun S, Morel H, Souquet P-J, et al. Staging of nutrition disorders in non-small-cell lung cancer patients: utility of skeletal muscle mass assessment. J Cachexia Sarcopenia Muscle. 2019;10:782-93.

21. Dell'Aquila E, Cremolini C, Zeppola T, et al. Prognostic and predictive role of neutrophil/lymphocytes ratio in metastatic colorectal cancer: a retrospective analysis of the TRIBE study by GONO. Ann Oncol. 2018;29:924-30.

22. Steinberg J, Erlichman C, Gadalla T, Fine S, Wong A. Prognostic factors in patients with metastatic colorectal cancer receiving 5-fluorouracil and folinic acid. Eur J Cancer. 1992;28A:1817-20.

23. Murphy RA, Mourtzakis M, Chu QSC, Baracos VE, Reiman T, Mazurak VC. Nutritional intervention with fish oil provides a benefit over standard of care for weight and skeletal muscle mass in patients with nonsmall cell lung cancer receiving chemotherapy. Cancer. 2011;117:1775-822.

24. Mouri T, Naito T, Morikawa A, et al. Promotion of behavioral change and the impact on quality of life in elderly patients with advanced cancer: a physical activity intervention of the multimodal nutrition and exercise treatment for advanced cancer program. Asia Pac J Oncol Nurs. 2018;5:383-90.

25. Zopf Y, Schink K, Reijic D, et al. Assessing cachexia in older patients: different definitions - but which one is the most practical for clinical routine? Arch Gerontol Geriatr. 2020;86:103943. 